\title{
Retraction Note to: Antiamoebic activity of synthetic tetrazoles against Acanthamoeba castellanii belonging to T4 genotype and effects of conjugation with silver nanoparticles
}

\author{
Areeba Anwar ${ }^{1} \cdot$ Yim Pei Yi $^{1} \cdot$ Itrat Fatima ${ }^{2} \cdot$ Khalid Mohammed Khan $^{2,3} \cdot$ Ruqaiyyah Siddiqui $^{4}$. \\ Naveed Ahmed Khan ${ }^{4}$. Ayaz Anwar ${ }^{1}$
}

Published online: 24 January 2022

○) Springer-Verlag GmbH Germany, part of Springer Nature 2022

\section{Retraction Note to: Parasitol Res (2020) 119:1943-1954 https://doi.org/10.1007/s00436-020-06694-4}

The authors have retracted this article because anomalies have been identified in Figures $2 b$ and $2 c$. The authors have repeated their study and have submitted a new manuscript for peer review. All authors agree with this retraction.

Publisher's note Springer Nature remains neutral with regard to jurisdictional claims in published maps and institutional affiliations.

The original article can be found online at https://doi.org/10.1007/ s00436-020-06694-4.

Ayaz Anwar

ayazanwarkk@yahoo.com

1 Department of Biological Sciences, School of Science and Technology, Sunway University, 47500 Subang Jaya, Selangor, Malaysia

2 H. E. J. Research Institute of Chemistry, International Center for Chemical and Biological Sciences, University of Karachi, Karachi 75270, Pakistan

3 Department of Clinical Pharmacy, Institute for Research and Medical Consultations (IRMC), Imam Abdulrahman Bin Faisal University, Dammam 31441, Saudi Arabia

4 Department of Biology, Chemistry and Environmental Sciences, College of Arts and Sciences, American University of Sharjah, Sharjah 26666, United Arab Emirates 